\title{
LOCAL ANOMALIES AROUND THE THIRD PEAK IN THE CMB ANGULAR POWER SPECTRUM OF WMAP 7-YEAR DATA
}

\author{
Kyeong Yeon Ko ${ }^{1,3}$, Chan-Gyung Park ${ }^{2}$, And Jai-Chan Hwang ${ }^{3}$ \\ ${ }^{1}$ Korea Astronomy and Space Science Institute, Daejeon, Korea \\ 2 Division of Science Education and Institute of Fusion Science, Chonbuk National University, Jeonju, Korea \\ ${ }^{3}$ Department of Astronomy and Atmospheric Sciences, Kyungpook National University, Daegu, Korea \\ E-mail : kyeongyeon.ko@gmail.com,parkc@jbnu.ac.kr,jchan@knu.ac.kr \\ (Received February 1, 2013; Revised March 20, 2013; Accepted March 21, 2013)
}

\begin{abstract}
We estimate the power spectra of the cosmic microwave background radiation (CMB) temperature anisotropy in localized regions of the sky using the Wilkinson Microwave Anisotropy Probe (WMAP) 7 -year data. We find that the north and south hat regions at high Galactic latitude $\left(|b| \geq 30^{\circ}\right)$ show an anomaly in the power spectrum amplitude around the third peak, which is statistically significant up to $3 \sigma$. We try to explain the cause of the observed anomaly by analyzing the low Galactic latitude $\left(|b|<30^{\circ}\right)$ regions where the galaxy contamination is expected to be stronger, and the regions weakly or strongly dominated by WMAP instrument noise. We also consider the possible effect of unresolved radio point sources. We find another but less statistically significant anomaly in the low Galactic latitude north and south regions whose behavior is opposite to the one at high latitude. Our analysis shows that the observed north-south anomaly at high latitude becomes weaker on regions with high number of observations (weak instrument noise), suggesting that the anomaly is significant at sky regions that are dominated by the WMAP instrument noise. We have checked that the observed north-south anomaly has weak dependences on the bin-width used in the power spectrum estimation, and on the Galactic latitude cut. We also discuss the possibility that the detected anomaly may hinge on the particular choice of the multipole bin around the third peak. We anticipate that the issue of whether or not the anomaly is intrinsic one or due to WMAP instrument noise will be resolved by the forthcoming Planck data.
\end{abstract}

Key words : cosmology: cosmic microwave background - cosmology: observations — methods: data analysis

\section{INTRODUCTION}

The CMB provides a wealth of information on the history of the Universe. The thermal blackbody nature of the CMB energy spectrum is now considered as a firm evidence of the hot big bang scenario for the beginning of the Universe (Alpher \& Herman 1948; Dicke et al. 1965; Penzias \& Wilson 1965). The existence of large-scale structures in the Universe also implies that there were primordial density perturbations as seeds for structure formation. It was expected that these inhomogeneities would leave their imprint on the CMB as minute temperature anisotropy (Sachs \& Wolfe 1967; Peebles \& Yu 1970; Bond \& Efstathiou 1987). The CMB anisotropy was discovered by the Cosmic Background Explorer (COBE) Differential Microwave Radiometers experiment (Smoot et al. 1992) and has been confirmed by many ground-based and balloon-borne experiments (see Hu \& Dodelson 2002; Scott \& Smoot 2006, for reviews and references therein).

Recently, the WMAP has opened a new window to

Corresponding Author: C.-G. Park the precision cosmology by measuring the CMB temperature anisotropy and polarization with high resolution and sensitivity (Bennett et al. 2003; Jarosik et al. 2011). For every data release, the WMAP team presented their estimation of the angular power spectra for temperature and polarization anisotropy (Hinshaw et al. 2003, 2007; Page et al. 2003, 2007; Nolta et al. 2009; Larson et al. 2011). By comparing the measured CMB power spectra with theoretical predictions, the WMAP team determined the cosmological parameters with a few percent precision (Spergel et al. 2003, 2007; Komatsu et al. 2009, 2011), and found that the observed CMB fluctuations are consistent with predictions of the concordance $\Lambda \mathrm{CDM}$ model with scaleinvariant adiabatic fluctuations generated during the inflationary epoch (Spergel et al. 2003; Peiris et al. 2003; Komatsu et al. 2011). Recent ground-based and balloon-borne experiments that have performed CMB power spectrum measurement and cosmological parameter estimation include the South Pole Telescope (SPT; Keisler et al. 2011), the QUaD experiment (Brown et al. 2009), the Arcminute Cosmology Bolometer Array Receiver (ACBAR; Reichardt et al. 2009), the Cosmic 
Background Imager (CBI; Mason et al. 2003), the Atacama Cosmology Telescope (ACT; Fowler et al. 2010), the Degree Angular Scale Interferometer (DASI; Carlstrom et al. 2003), BOOMERANG (Jones et al. 2006), Archeops (Benoît et al. 2003), and MAXIMA (Lee et al. 2001).

In CMB data analysis, two-point statistics (i.e., correlation function and power spectrum) have been widely used. In particular, the relation between the CMB angular power spectrum and cosmological physics is well understood, and tight constraints on cosmological parameters can be directly obtained by comparing the measured power spectrum with the corresponding theoretical prediction. Therefore, an accurate estimation of the angular power spectrum from observed CMB maps is essential. Efficient techniques to measure the angular power spectrum from CMB temperature fluctuations with incomplete sky coverage have been constantly developed (e.g., Górski 1994; Tegmark 1997; Bond et al. 1998; Oh et al. 1999; Szapudi et al. 2001; Wandelt et al. 2001; Hansen et al. 2002; Hivon et al. 2002; Mortlock et al. 2002; Hinshaw et al. 2003; Wandelt et al. 2003; Chon et al. 2004; Efstathiou 2004; Eriksen et al. 2004a; Wandelt et al. 2004; Brown et al. 2005; Polenta et al. 2005; Fay et al. 2008; Dahlen \& Simons 2008; Das et al. 2009; Mitra et al. 2009; Ansari et al. 2010; Chiang \& Chen 2012).

Until now, the analysis of WMAP/CMB data has been mainly made using the whole sky area except for strongly contaminated regions (Hinshaw et al. 2003, 2007; Nolta et al. 2009; Larson et al. 2011; Saha et al. 2006, 2008; Souradeep et al. 2006; Eriksen et al. 2007a; Samal et al. 2010; Basak \& Delabrouille 2012). On the other hand, there has been less studies on the power spectrum measurement on partial regions of the sky (e.g., Eriksen et al. 2004b; Hansen et al. 2004a,b; Ansari et al. 2010; Yoho et al. 2011; Chiang \& Chen 2012). In particular, Yoho et al. (2011) detected a degree-scale anomaly around the first acoustic peak of the CMB angular power spectrum measured on a small patch of the north ecliptic sky. In this work, we measure the angular power spectra from the WMAP 7-year temperature anisotropy data set on some specified regions of the sky. We find that at high Galactic latitude there is a north-south anomaly or asymmetry in the power amplitude around the third peak of the angular power spectrum, which is the main result of this paper. Our result differs from the well-known hemispherical asymmetry in the angular power spectrum and the genus topology at large angular scales (Park 2004; Eriksen et al. 2004b,c, 2007b; Hansen et al. 2004b; Bernui 2008; Hansen et al. 2009; Hoftuft et al. 2009).

This paper is organized as follows. In Section 2, we describe the method used to measure the angular power spectrum. The application of the method to WMAP 7-year data and the comparison with the WMAP team results are shown in Section 3. In Section 4 , we measure the power spectra on various sky regions, and present our discovery of the north-south anomaly around the third peak of the angular power spectrum at high Galactic latitude regions. We also discuss the potential origin of such anomaly. Section 5 contains the conclusions. Throughout this work, we have used the HEALPix software (Górski et al. 1999, 2005) to measure the pseudo angular power spectra and to generate the WMAP mock data sets.

\section{ANGULAR POWER SPECTRUM ESTI- MATION METHOD}

In this section we briefly review how the angular power spectrum is measured from the observed CMB temperature anisotropy maps. In what follows, we do not consider the CMB polarization.

In the ideal situation where the temperature fluctuations are observed on the whole sky, we can expand the temperature distribution $T(\mathbf{n})$ in terms of spherical harmonics as

$$
T(\mathbf{n})=\sum_{l=2}^{\infty} \sum_{m=-l}^{l} a_{l m} Y_{l m}(\mathbf{n}),
$$

where $\mathbf{n}$ denotes the angular position on the sky, $Y_{l m}(\mathbf{n})$ is the spherical harmonic basis function, and the monopole $(l=0)$ and dipole $(l=1)$ components have been neglected. From the orthogonality condition of spherical harmonics, the coefficients $a_{l m}$ can be obtained from

$$
a_{l m}=\int d \Omega T(\mathbf{n}) Y_{l m}^{*}(\mathbf{n}),
$$

where $d \Omega$ denotes the differential solid angle on the sky. If the temperature anisotropy is statistically isotropic, then the variance of the harmonic coefficients $a_{l m}$ is independent of $m$ and the angular power spectrum $C_{l}$ is given as the ensemble average of the two-point product of harmonic coefficients,

$$
\left\langle a_{l m} a_{l^{\prime} m^{\prime}}^{*}\right\rangle=C_{l} \delta_{l l^{\prime}} \delta_{m m^{\prime}},
$$

where the bracket represents the ensemble average and $\delta_{l l^{\prime}}$ is the Kronecker delta symbol $\left(\delta_{l l^{\prime}}=1\right.$ for $l=l^{\prime}$, and 0 for $\left.l \neq l^{\prime}\right)$. For a Gaussian temperature distribution, all the statistical information is included in the two-point statistics, i.e., in the angular power spectrum $C_{l}$.

For a single realization of temperature fluctuations on the sky, the ensemble average is replaced with the simple average of independent modes belonging to the same multipole $l$, and the angular power spectrum becomes

$$
C_{l}^{\mathrm{sky}}=\frac{1}{2 l+1} \sum_{m=-l}^{l}\left|a_{l m}\right|^{2},
$$

with uncertainty due to cosmic variance

$$
\Delta C_{l}=\sqrt{\frac{2}{2 l+1}} C_{l} .
$$


In practice, however, we usually exclude some portions of the sky area where the contamination due to Galactic emission and strong radio point sources is expected to affect the statistics of the CMB anisotropy significantly. The limited angular resolution, instrument noise, and finite pixels of the processed map also prevent us from using Eqs. 1-5, which are valid only in the ideal situation. The incomplete sky coverage with an arbitrary geometry and the limited instrumental performance break the orthogonality relation between spherical harmonic functions. Thus, measuring angular power spectrum from observational data is more complicated.

There are two common ways of measuring the angular power spectrum from observed CMB data with incomplete sky coverage. One is the maximum likelihood estimation method based on the Bayesian theorem (Bond et al. 1998; Tegmark 1997); the other is the pseudo power spectrum method that applies the Fourier or harmonic transformation directly (Hivon et al. 2002). For the latter, Hivon et al. (2002) developed the so called MASTER (Monte Carlo Apodised Spherical Transform Estimator) method, which estimates the angular power spectrum from high resolution CMB data. For mega-pixelized data sets as those of WMAP or Planck (Planck Collaboration 2011a), the MASTER method is faster and more efficient than the maximum likelihood approach.

We denote the pseudo angular power spectrum as $\tilde{C}_{l}$ to distinguish it from the true angular power spectrum $C_{l}$ which we try to estimate. The two quantities differ from each other because of the incomplete sky coverage, the limited angular resolution and pixel size, and the instrument noise; they are related by (Hansen et al. 2002; Hivon et al. 2002)

$$
\left\langle\tilde{C}_{l}\right\rangle=\sum_{l^{\prime}} M_{l l^{\prime}} F_{l^{\prime}} B_{l^{\prime}}^{2}\left\langle C_{l^{\prime}}\right\rangle+\left\langle\tilde{N}_{l}\right\rangle
$$

where $B_{l}$ is the beam transfer function, $F_{l}$ the pixel transfer function due to finite pixel size of the CMB map, $\left\langle\tilde{N}_{l}\right\rangle$ the average pseudo noise power spectrum due to the instrument noise, and $M_{l l^{\prime}}$ the mode-mode coupling matrix that incorporates all the effects due to incomplete sky coverage. The mode-mode coupling matrix is written as

$$
M_{l_{1} l_{2}}=\frac{2 l_{2}+1}{4 \pi} \sum_{l_{3}}\left(2 l_{3}+1\right) \mathcal{W}_{l_{3}}\left(\begin{array}{ccc}
l_{1} & l_{2} & l_{3} \\
0 & 0 & 0
\end{array}\right)^{2},
$$

where $\mathcal{W}_{l}$ is the power spectrum of the window function for incomplete sky coverage (see Section 3.2), and the last factor on the right-hand side enclosed with the parenthesis represents the Wigner 3- $j$ symbol. We use Fortran and Mathematica* to calculate the Wigner 3$j$ symbols; see also Hivon et al. (2002) and Brown et

*Wolfram Research Inc., http://wolfram.com/mathematica al. (2005) for the numerical computation of the Wigner 3 - $j$ symbols.

To reduce the statistical variance of the measured angular power spectrum due to cosmic variance and instrument noise, we average powers within the appropriate l-bins. According to Hivon et al. (2002), the binning operator is defined as

$$
P_{b l}= \begin{cases}\frac{1}{2 \pi} \frac{l(l+1)}{l_{\text {low }}^{(b+1)}-l_{\text {low }}^{(b)},}, & \text { if } 2 \leq l_{\text {low }}^{(b)} \leq l<l_{\text {low }}^{(b+1)} \\ 0, & \text { otherwise. }\end{cases}
$$

The reciprocal operator is

$$
Q_{l b}= \begin{cases}\frac{2 \pi}{l(l+1)}, & \text { if } 2 \leq l_{\text {low }}^{(b)} \leq l<l_{\text {low }}^{(b+1)} \\ 0, & \text { otherwise }\end{cases}
$$

where $b$ is the bin index. For comparison, we use exactly the same $l$-binning adopted by the WMAP team ( $b$ runs from 1 to 45, Larson et al. 2011) The true power spectrum can be estimated from

$$
\hat{C}_{b}=\left(K^{-1}\right)_{b b^{\prime}} P_{b^{\prime} l}\left(\tilde{C}_{l}-\left\langle\tilde{N}_{l}\right\rangle\right),
$$

where

$$
K_{b b^{\prime}}=P_{b l} M_{l l^{\prime}} F_{l^{\prime}} B_{l^{\prime}}^{2} Q_{l^{\prime} b^{\prime}}
$$

is a kernel matrix that describes the mode-mode couplings due to the survey geometry, finite resolution and pixel size, and the binning process.

Ideally, the average pseudo noise power spectrum $\left\langle\tilde{N}_{l}\right\rangle$ can be estimated by a Monte Carlo simulation that mimics the instrument noise. However, because of statistical fluctuations in the noise power spectra, it is rather difficult to obtain an accurate estimation of the power contribution due to instrument noise from a single observed data. In our analysis we evade this problem by measuring the cross-power spectra between different channel data sets, in the same way as done by the WMAP team (see the next section).

\section{APPLICATION TO WMAP 7-YEAR DATA}

\subsection{WMAP 7-Year Data}

The WMAP mission was designed to construct full sky CMB temperature and polarization maps with high accuracy, precision, and reliability (Bennett et al. 2003). The WMAP instrument has 10 differencing assemblies (DAs) spanning five frequency bands from 23 to $94 \mathrm{GHz}$ : one DA each at $23 \mathrm{GHz}(\mathrm{K} 1)$ and $33 \mathrm{GHz}$ (Ka1), two DAs each at $41 \mathrm{GHz}(\mathrm{Q} 1, \mathrm{Q} 2)$ and $61 \mathrm{GHz}$ (V1, V2), and four DAs at $94 \mathrm{GHz}$ (W1, W2, W3, W4), with $0.82,0.62,0.49,0.33$, and 0.21 FWHM beam widths, respectively. In this work, we use the foreground-reduced WMAP 7-year temperature fluctuation maps in HEALPix format, with $N_{\text {side }}=512$ (resolution $9 ; \mathrm{r} 9$ ). The total number of pixels of each map is $12 \times N_{\text {side }}^{2}=3,145,728$. Following the WMAP 
team analysis, we use only $\mathrm{V}$ and $\mathrm{W}$ band maps to reduce any possible contamination due to Galactic foregrounds. We use the WMAP beam transfer function $\left(B_{l}\right)$ and the number of observations $\left(N_{\text {obs }}\right)$ for each DA. The data sets used in this work are available on the NASA's Legacy Archive for Microwave Background Data Analysis (LAMBDA) ${ }^{\dagger}$.

\subsection{Weighting Schemes}

The window function assigns a weight to the temperature fluctuation on each pixel before the harmonic transformation is performed. In our analysis, the window function maps have been produced based on two weighting schemes; the uniform weighting scheme, and the inverse-noise weighting scheme (Hinshaw et al. 2003). The WMAP team provides mask maps which assign unity to pixels used in the analysis, and zero to pixels excluded to avoid foreground contamination. We use the KQ85 mask with resolution 9, that includes about $78.3 \%$ of the whole sky area. The mask excludes the Galactic plane region with strong Galactic emission, and the circular areas with 0.6 radius centered on strong radio point sources. The uniform weighting scheme uses the mask map as the window function directly,

$$
W(p)=M(p),
$$

where $M(p)$ denotes a mask map on a pixel $p$ on the sky. The power spectrum at high- $l$ regions is usually dominated by instrument noise. In the WMAP data the noise level on each pixel is modeled by

$$
\sigma(p)=\frac{\sigma_{0}}{\sqrt{N_{\mathrm{obs}}(p)}},
$$

where $N_{\text {obs }}(p)$ is the number of observations per pixel $p$, and $\sigma_{0}$ the global noise level $\left(\sigma_{0}=3.319,2.955,5.906\right.$, $6.572,6.941,6.778 \mathrm{mK}$ for $\mathrm{V} 1, \mathrm{~V} 2, \mathrm{~W} 1, \mathrm{~W} 2, \mathrm{~W} 3, \mathrm{~W} 4$ DAs, respectively). To reduce the effect of instrument noise, the inverse-noise weighting scheme is defined as the product of the mask map and the map with the number of observations,

$$
W(p)=M(p) N_{\text {obs }}(p) .
$$

The window function of the $94 \mathrm{GHz}$ W4 frequency channel (DA) is shown in Fig. 1, together with a histogram of the number of observations. Although the WMAP team applies the uniform weighting scheme for $l<600$ and the inverse-noise weighting scheme for $l>600$ (Larson et al. 2011), in this work we simply apply a single weighting scheme over the whole range of $l$ considered $(2 \leq l \leq 1200)$ during the power spectrum estimation, and compare the results based on the two weighting schemes.

\footnotetext{
${ }^{\dagger}$ http://lambda.gsfc.nasa.gov
}

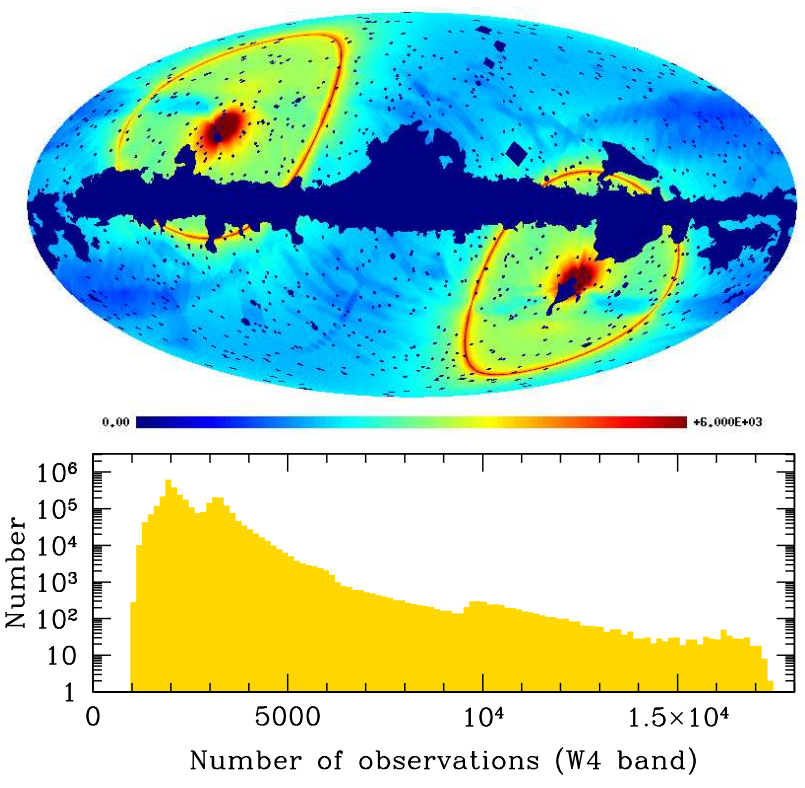

Fig. 1.- (Top) A map of the number of observations $N_{\text {obs }}(p)$ from the WMAP 7-year W4 frequency channel (DA), multiplied by the KQ85 mask map $M(p)$ [see Eq. 12]. Dark blue regions correspond to zero values, and represents areas excluded by the KQ85 mask map, while dark red regions denote values exceeding $N_{\text {obs }} \geq 6000$. The Mollweide projection in Galactic coordinates is used to display this map, where the Galactic center is located at the center, the Galactic longitude increases from center to left $\left(l=0^{\circ}-\right.$ $\left.180^{\circ}\right)$ and from right to center $\left(l=180^{\circ}-360^{\circ}\right)$, and the Galactic latitude increases from bottom to top $\left(b=-90^{\circ}-\right.$ $\left.+90^{\circ}\right)$. The regions with large number of observations correspond to the ecliptic poles. (Bottom) Histogram of the number of observations at the same frequency channel.

\subsection{Angular Power Spectrum Measured from WMAP Data}

In the WMAP team data analysis, the power spectrum at low multipoles $(l \leq 32)$ was measured by a Blackwell-Rao estimator applied to a chain of Gibbs samples obtained from the foreground-cleaned CMB map, and the power spectrum at high multipoles $(32<$ $l \leq 1200)$ by the MASTER pseudo power spectrum estimation method (Larson et al. 2011). Throughout this work, the angular power spectrum over the whole $l$ range $(2 \leq l \leq 1200)$ is measured with the pseudo power spectrum estimation method, because our primary attention is on the high- $l$ region where the peaks are located. Thus, as shown below, our results at low multipoles are somewhat different from the WMAP team results.

The WMAP V and $\mathrm{W}$ bands have two (V1 and V2) and four (W1, W2, W3, and W4) separate frequency channels (DAs), respectively. Using ANAFAST (from the HEALPix package), we measured 15 pseudo cross power spectra $\left(\tilde{C}_{l}\right)$ for different channel combinations 
(V1W1, V1W2, and so on) based on the uniform and inverse-noise weighting schemes. During the production of each pseudo cross power spectrum, ANAFAST estimates two separate sets of harmonic coefficients for each DA using Eq. 2, where $T(\mathbf{n})$ is now replaced by $W(\mathbf{n}) T(\mathbf{n})$ and $W(\mathbf{n})$ is the window function defined in Eqs. 10 and 12 for the corresponding DA. We set the maximum multipole to $l=1200$.

For each channel combination, we calculate the mode-mode coupling matrix $M_{l l^{\prime}}$ and the kernel matrix $K_{b b^{\prime}}$ using the beam and pixel transfer functions, and the same $l$-binning as defined by the WMAP team (Larson et al. 2011). For $\mathcal{W}_{l}$ in Eq. 7 we use the cross power spectrum of the window functions for the corresponding DA combination, which is obtained from ANAFAST by inserting the window functions at two different frequency channels as the input data. The squared beam transfer function $B_{l}^{2}$ appearing in Eq. 6 is also modified as the product of beam transfer functions from the two different frequency channels.

The final binned cross power spectrum $\hat{C}_{b}$ for each channel combination is obtained from Eq. 8, neglecting the term for the average noise power spectrum $\left\langle\tilde{N}_{l}\right\rangle$. Since the properties of the instrument noise for each channel are independent, the process of crosscorrelation statistically suppresses the contribution of the instrument noise to the power spectrum estimation. This technique has the advantage that our power spectrum estimator is not biased by noise if the noise in the two independent channels is uncorrelated; the crosscorrelation between the uncorrelated noise vanishes statistically. Thus, the measured cross-power spectra are independent of instrument noise from the individual channels (see Hinshaw et al. 2003). Note that the selfcombination of each frequency channel data (as V1V1, $\mathrm{V} 2 \mathrm{~V} 2$, W1W1) is not considered during the analysis, because it is needed to subtract the contribution of the noise power from the measured auto-power spectrum and it is a difficult task to estimate precisely the noise power from a single observed map (see the discussion at the end of Section 2). For each binned cross power spectrum we subtract the contribution due to unresolved radio point sources expected in the WMAP temperature anisotropy maps by using the information presented in Nolta et al. (2009) and Larson et al. (2011). The amplitude of the binned power spectrum due to unresolved point sources can be written as

$$
C_{\mathrm{ps}, b}^{i}=A_{\mathrm{ps}} P_{b l} S_{l}^{i} .
$$

Here $S_{l}^{i}$ is the point-source spectral function given by

$$
S_{l}^{i}=r\left(\nu_{k}\right) r\left(\nu_{k^{\prime}}\right)\left(\frac{\nu_{k} \nu_{k^{\prime}}}{\nu_{\mathrm{Q}}^{2}}\right)^{\beta},
$$

where $\nu_{k}$ and $\nu_{k^{\prime}}$ denote the individual frequency channel belonging to the $i$-th channel combination, $r\left(\nu_{k}\right)$ is a conversion factor from antenna to thermodynamic temperature, $\nu_{\mathrm{Q}}=40.7 \mathrm{GHz}$ is the Q-band central frequency, $10^{3} A_{\mathrm{ps}}=9.0 \pm 0.7 \mu \mathrm{K}^{2}$, and $\beta=-2.09$ (see also Huffenberger et al. 2006; Souradeep et al. 2006).

To combine these cross power spectra into a single angular power spectrum, we need Monte Carlo simulations with similar properties of the WMAP observational data. Using SYNFAST (from the HEALPix package) and assuming a concordance flat $\Lambda \mathrm{CDM}$ model with parameters $\Omega_{b} h^{2}=0.02260, \Omega_{c} h^{2}=$ $0.1123, \Omega_{\Lambda}=0.728, n_{s}=0.963, \tau=0.087, \Delta_{\mathcal{R}}^{2}(k=$ $\left.0.002 \mathrm{Mpc}^{-1}\right)=2.441 \times 10^{-9}$ (Table 14 of Komatsu et al. 2011), we made 1000 mock data sets that mimic the WMAP beam resolution and instrument noise for each channel. We used CAMB to obtain the theoretical model power spectrum (Lewis et al. 2000). We assumed that the temperature fluctuations and instrument noise follow a Gaussian distribution. In the simulations we do not consider any effect due to residual foreground contamination. We have analyzed the WMAP mock data sets in the same way as the real data set.

Before combining the cross power spectra into a single power spectrum, each cross power spectrum has been averaged within $l$-bins, to reduce the statistical fluctuations due to cosmic variance and instrument noise. We use the same $l$-bins defined by the WMAP 7 -year data analysis (Larson et al. 2011). Thus, when we measure the power spectrum from the WMAP data with a sky fraction much smaller than the area enclosed by the KQ85 mask, the measured powers at low- and high-l regions become uncertain and fluctuate significantly with strong cross-correlations between adjacent bins (see below). We average the 15 binned cross power spectra into a single angular power spectrum by applying an algorithm similar to that used in the WMAP first year data analysis (Hinshaw et al. 2003). For each $l$-bin, we construct a covariance matrix defined as

$$
\left(\Sigma_{\text {full }}\right)_{b}^{i j}=\left\langle\left[C_{b}^{i}-\bar{C}_{b}^{i}\right]\left[C_{b}^{j}-\bar{C}_{b}^{j}\right]\right\rangle+\left(\Sigma_{\mathrm{src}}\right)_{b}^{i j},
$$

where $i$ and $j$ denote the cross combination of the WMAP frequency channels (V1V2, V1W1, W1W3, and so on $), b$ is the binning index $(b=1, \ldots, 45), C_{b}^{i}$ is the measured cross power spectrum for the channel combination $i, \bar{C}_{b}^{i}$ is the ensemble averaged cross power spectrum for the same channel combination expected in the concordance $\Lambda \mathrm{CDM}$ model (we have used 1000 WMAP mock data sets to estimate the ensemble averaged quantities), and

$$
\left(\Sigma_{\mathrm{src}}\right)_{b}^{i j}=\left(P_{b l} S_{l}^{i}\right)\left(P_{b l^{\prime}} S_{l^{\prime}}^{j}\right) \sigma_{\mathrm{src}}^{2}
$$

is the covariance matrix between errors expected to be caused during the subtraction of powers due to unresolved point sources. We use $\sigma_{\mathrm{src}}^{2}=\left(\delta A_{\mathrm{ps}}\right)^{2}=$ $\left(0.0007 \mu \mathrm{K}^{2} \mathrm{sr}\right)^{2}$. The final combined angular power spectrum is obtained from

$$
\hat{C}_{b}=\frac{\sum_{i=1}^{15} \sum_{j=i}^{15} \hat{C}_{b}^{i}\left(\Sigma_{\mathrm{full}}^{-1}\right)_{b}^{i j}}{\sum_{i=1}^{15} \sum_{j=i}^{15}\left(\Sigma_{\mathrm{full}}^{-1}\right)_{b}^{i j}}
$$



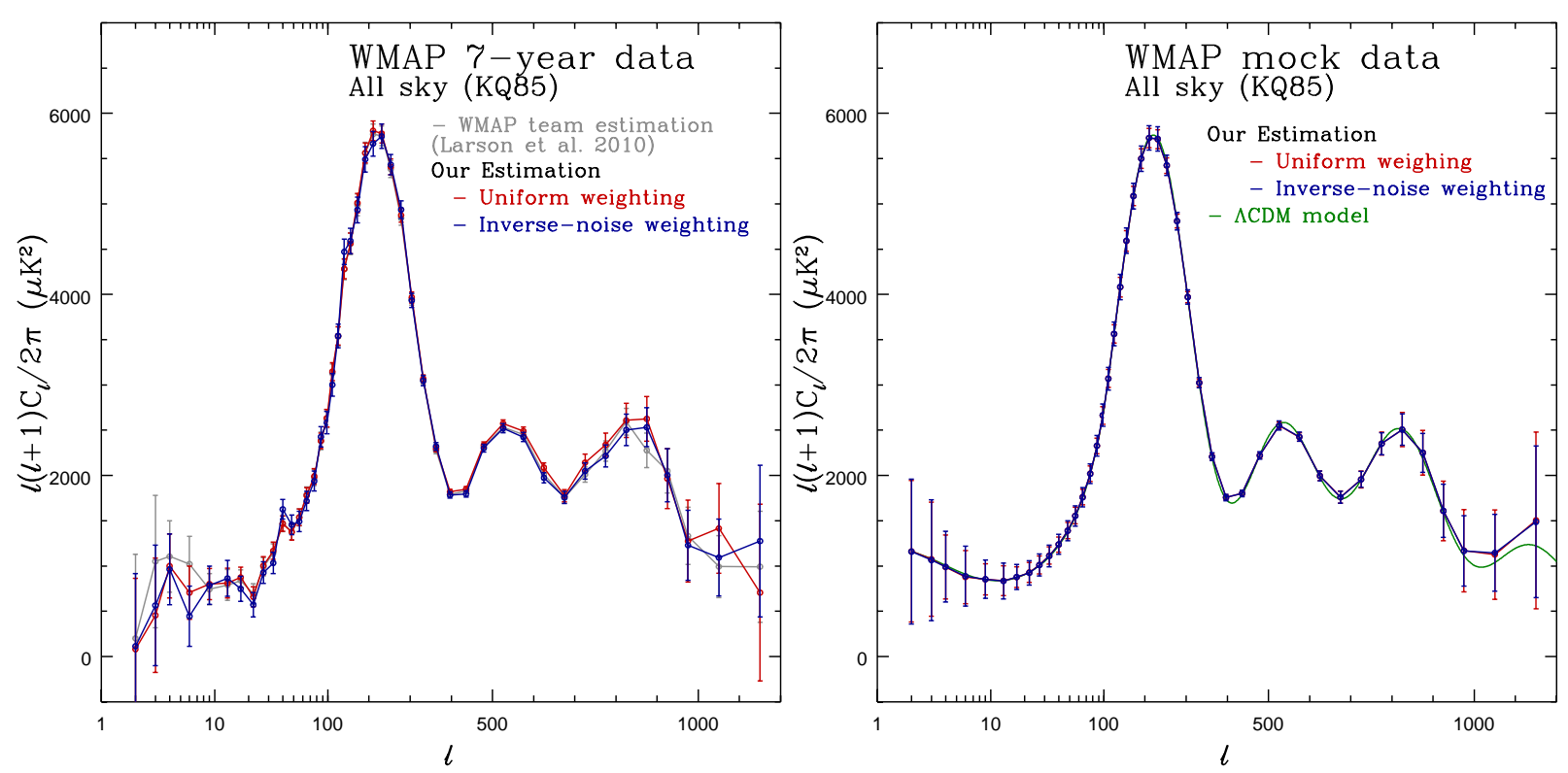

Fig. 2.- (Left) Angular power spectra measured from the WMAP 7-year temperature anisotropy maps. Our results are shown as red and blue curves with dots and error bars for uniform and inverse-noise weighting schemes, respectively. The sky area (78.27\% of the whole sky) defined by the KQ85 mask map has been used. For comparison, the WMAP team result is also shown as the grey curve with dots and error bars (Larson et al. 2011). (Right) Averaged angular power spectra measured from 1000 WMAP mock data sets consistent with the concordance $\Lambda$ CDM model (Komatsu et al. 2011). The red color denotes the result based on the uniform weighting scheme, while the blue is based on the inverse-noise weighting scheme. The power spectrum of the assumed concordance $\Lambda$ CDM model is shown with the green curve.

To estimate the uncertainty of the combined power spectrum $\hat{C}_{b}$, we obtain 1000 combined power spectra from the mock data sets $\left(\hat{C}_{b}^{\text {sim }}\right)$, and consider their average and standard deviation at each bin. The latter is used as the uncertainty (or error bar) for the measured combined power spectrum. By comparing the theoretical power spectrum with the average one obtained from the simulation data sets, we can check whether or not our power spectrum measurement algorithm works correctly.

The left panel of Fig. 2 shows the angular power spectra of the WMAP 7-year temperature maps measured with the uniform (red) and inverse-noise (blue dots with error bars) weighting schemes. We have used the sky area defined by the KQ85 mask that was also used by the WMAP team temperature analysis. For comparison, the WMAP team measurement is shown, i.e., grey dots with error bars (Larson et al. 2011). Our estimations of the angular power spectrum in both the uniform and inverse-noise weighting schemes are consistent with the WMAP team results. We note that our amplitude of the power spectrum at the 41th bin $(851 \leq l \leq 900)$ is slightly larger than the WMAP team estimation, but the two power spectra are statistically consistent with each other. However, our result is a bit different from the WMAP team result in the low- $l$ region, because the WMAP team applied a different estimation method (Blackwell-Rao estimator based on Gibbs sampling) in this low- $l$ region while we simply used the pseudo power spectrum estimation method over the whole $l$-range.

The right panel of Fig. 2 shows the averaged angular power spectra measured from 1000 mocks. The averaged power spectra based on both the uniform and inverse-noise weighting schemes are consistent with each other, and with the assumed theoretical power spectrum (green curve) within uncertainties, which demonstrates that our algorithm works correctly. However, our procedure has a slightly positive bias with respect to the true value in the last two bins at the highest multipoles, where the uncertainty due to finite pixel size is significantly large. Comparing the results based on the two weighting schemes, we can see that for the inverse-noise weighting scheme the sizes of the error bars are bigger (smaller) at lower (higher) multipoles.

\section{POWER SPECTRA OF VARIOUS LO- CAL SKY AREAS}

Here we present the angular power spectra measured on various local regions of the sky. The regions are defined based on simple criteria, such as Galactic latitude or number-of-observations. To define a partial sky region, we use the KQ85 mask map and the number of observations in the $\mathrm{W}$ band (W4 DA). By putting a limit on the Galactic latitude or on the number of observations, we have obtained several local regions with different characteristics. 


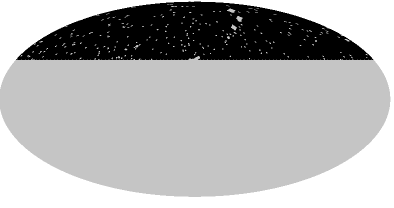

(a) High latitude, north hat with $b \geq 30^{\circ}$ $(24.11 \%)$

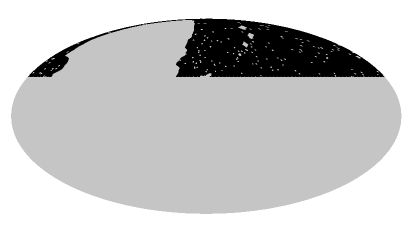

(e) North hat with high instrument noise $(15.20 \%)$

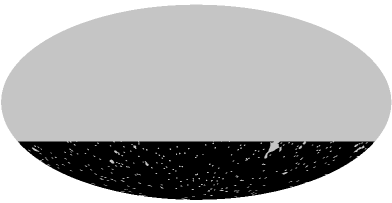

(b) High latitude, south hat with $b \leq-30^{\circ}$ $(23.98 \%)$

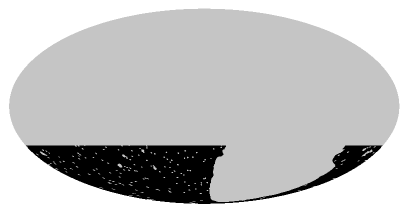

(f) South noise $(15.14 \%)$

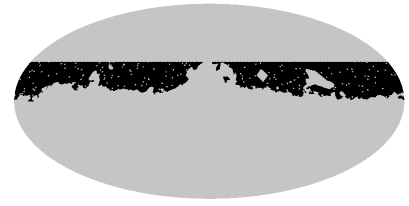

(c) Low latitude, north region with $0^{\circ}<b<30^{\circ}$ (15.15\%)

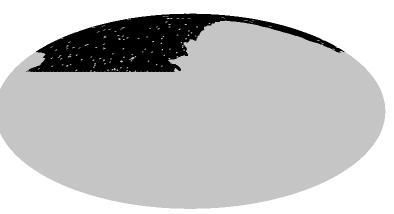

(g) North hat with low instrument noise $(13.05 \%)$

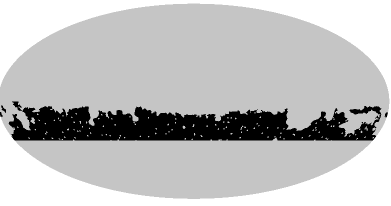

(d) Low latitude, south region with $-30^{\circ}<b<$ $0^{\circ}(15.03 \%)$

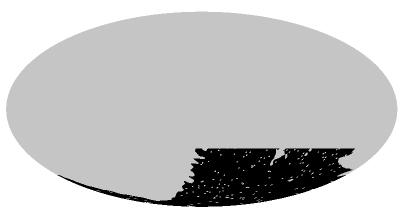

(h) South hat with low instrument noise $(13.01 \%)$

Fig. 3. - Mask maps of various local areas defined by applying the Galactic latitude cuts $[(a)-(d)]$ and the thresholds on the (smoothed) map of the number-of-observations $[(e)-(h)]$ on the KQ85 mask map. The black area corresponds to $M(p)=1$ while the grey area to $M(p)=0$. Each number between parenthesis indicates the fraction of the sky area with $M(p)=1$.

Fig. 3 summarizes the mask maps (or the window functions in the uniform weighting scheme) used in our power spectrum measurements. We estimate the angular power spectra on each local area using the corresponding mask map as a window function. In particular, the north $\left(b \geq 30^{\circ} ; b\right.$ is the Galactic latitude) and south hat $\left(b \leq-30^{\circ}\right)$ regions defined by a simple Galactic latitude cut show a difference in the power amplitude around the third peak of the angular power spectrum, which may represent a new anomaly (see Fig. 4 below). To assess the statistical significance of the observed anomaly, we compare the difference in the power spectrum amplitudes with the prediction of the fiducial $\Lambda$ CDM model. Then, we search for the origin of the anomaly by considering a residual Galaxy contamination, the WMAP instrument noise, and unresolved point sources.

\subsection{North and South Hat Regions}

Fig. 4 displays the angular power spectra measured on the north hat $(\mathrm{NH})$ and the south hat $(\mathrm{SH})$ regions. Each power spectrum has been estimated both in the uniform and inverse-noise weighting schemes separately. The overall features in the measured angular power spectra are similar to those of the power spectrum measured on the whole sky region with the KQ85 mask (grey curves). However, the amplitudes of the power spectrum around the third peak show opposite deviations in the $\mathrm{SH}$ and $\mathrm{NH}$ regions.

We can notice such a difference more dramatically in the middle panels of Fig. 4, where the difference of powers between the $\mathrm{SH}$ and $\mathrm{NH}$ are displayed for comparison. We have also estimated the average and the variance of the differences between $\mathrm{SH}$ and $\mathrm{NH}$ powers from 1000 WMAP mock data sets, shown as dark green dots with $1 \sigma$ (dark grey) and $3 \sigma$ (light grey) error bars. Since we have assumed homogeneity and isotropy of the Universe during the production of the WMAP mock data sets, the average of the differences is expected to be zero over the whole $l$-range. We note that in the third peak (that corresponds to the 40th bin; $801 \leq l \leq 850)$ the difference between $\mathrm{SH}$ and $\mathrm{NH}$ regions is statistically significant, since it is around $3 \sigma$. Both the histogram of the differences between $\mathrm{SH}$ and $\mathrm{NH}$ powers in the 40th bin measured from the mocks and the value measured from the WMAP data (vertical red dashed line) confirm this result (bottom panels of Fig. 4).

\subsection{Low Latitude Area}

The foreground contamination at high Galactic latitude regions like the $\mathrm{NH}$ and $\mathrm{SH}$ is generally expected to be small. Although the foreground model has been subtracted from the observed temperature fluctuations, and the sky region significantly contaminated by the Galactic emission has been excluded by the KQ85 mask, we can expect that the residual foreground emission at low latitude regions may be stronger than at high latitude regions (such as the $\mathrm{NH}$ and $\mathrm{SH}$ ). We estimated the angular power spectra in two separate regions at the Galactic latitude defined as low latitude north $\left(0^{\circ}<b<30^{\circ}\right)$ and south $\left(-30^{\circ}<b<0^{\circ}\right)$, 

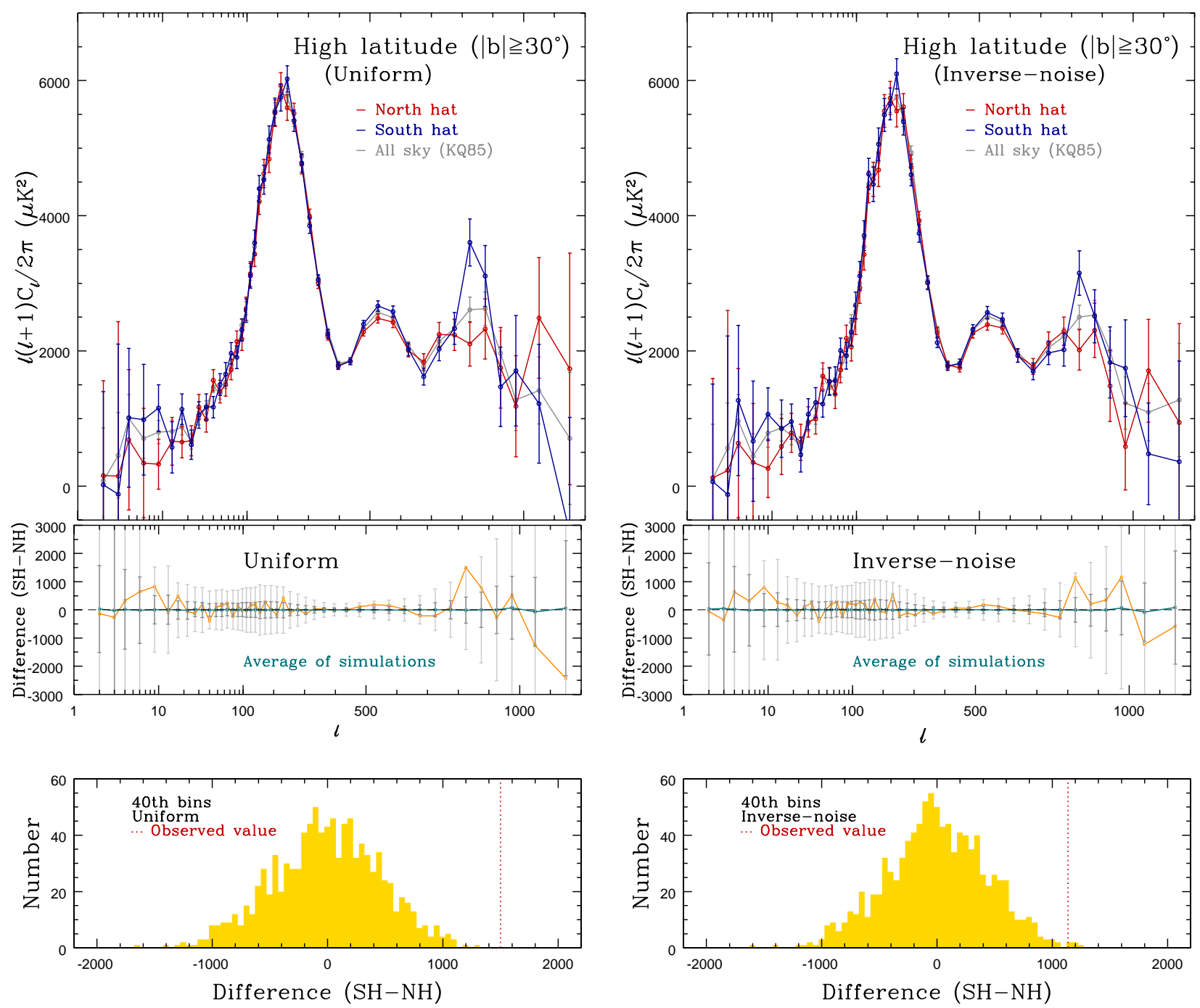

Fig. 4.- (Top) Angular power spectra measured on the NH and SH regions based on the uniform (left) and inverse-noise (right panel) weighting schemes. The power spectra measured on the $\mathrm{NH}\left(b \geq 30^{\circ}\right)$ are shown in red, while those on the $\mathrm{SH}$ $\left(b \leq-30^{\circ}\right)$ in blue. The grey color denotes the result measured on the whole sky enclosed by the KQ85 mask map. (Middle) Power differences between the SH and NH are shown (orange). The averaged difference estimated from 1000 WMAP mocks are shown as dark green dots with $1 \sigma$ (dark grey) and $3 \sigma$ (light grey) error bars. (Bottom) Histograms of the differences between $\mathrm{SH}$ and $\mathrm{NH}$ powers in the 40th bin that corresponds to $801 \leq l \leq 850$, measured from the mocks, for uniform (left) and inverse-noise (right panel) weighting schemes. The vertical red dashed lines indicate the difference values measured from the WMAP 7-year data.

where the Galactic plane region has been excluded by the KQ85 mask. The results are presented in Fig. 5, similarly as in Fig. 4.

The angular power spectra measured on sky regions contaminated by a residual Galactic foreground emission show different features from those seen in the $\mathrm{NH}$ and SH. The behavior of power spectrum amplitudes around the third peak is opposite to the case of the $\mathrm{NH}$ and $\mathrm{SH}$ regions. The power on the low latitude north region is larger than that on the south region, and the difference between north and south regions is more significant at the 41 th bin $(851 \leq l \leq 900)$. The difference is again statistically significant (i.e., a $3 \sigma$ deviation for the uniform weighting scheme), another anomaly found in this work. Although not shown here, if we consider the whole northern and southern hemispheres as two localized regions, then the anomalies at high and low Galactic latitudes are compensated and not noticeable. In addition, there are two more noticeable differences between the north and south regions in the second bin $(l=3)$ and in the last one $(1101 \leq l \leq 1200)$. For the latter, the measured difference exceeds $3 \sigma$ from the zero value for the inverse-noise weighting scheme (see also Table 1 below). Therefore, based on this comparison, it seems that the observed north-south anomaly 

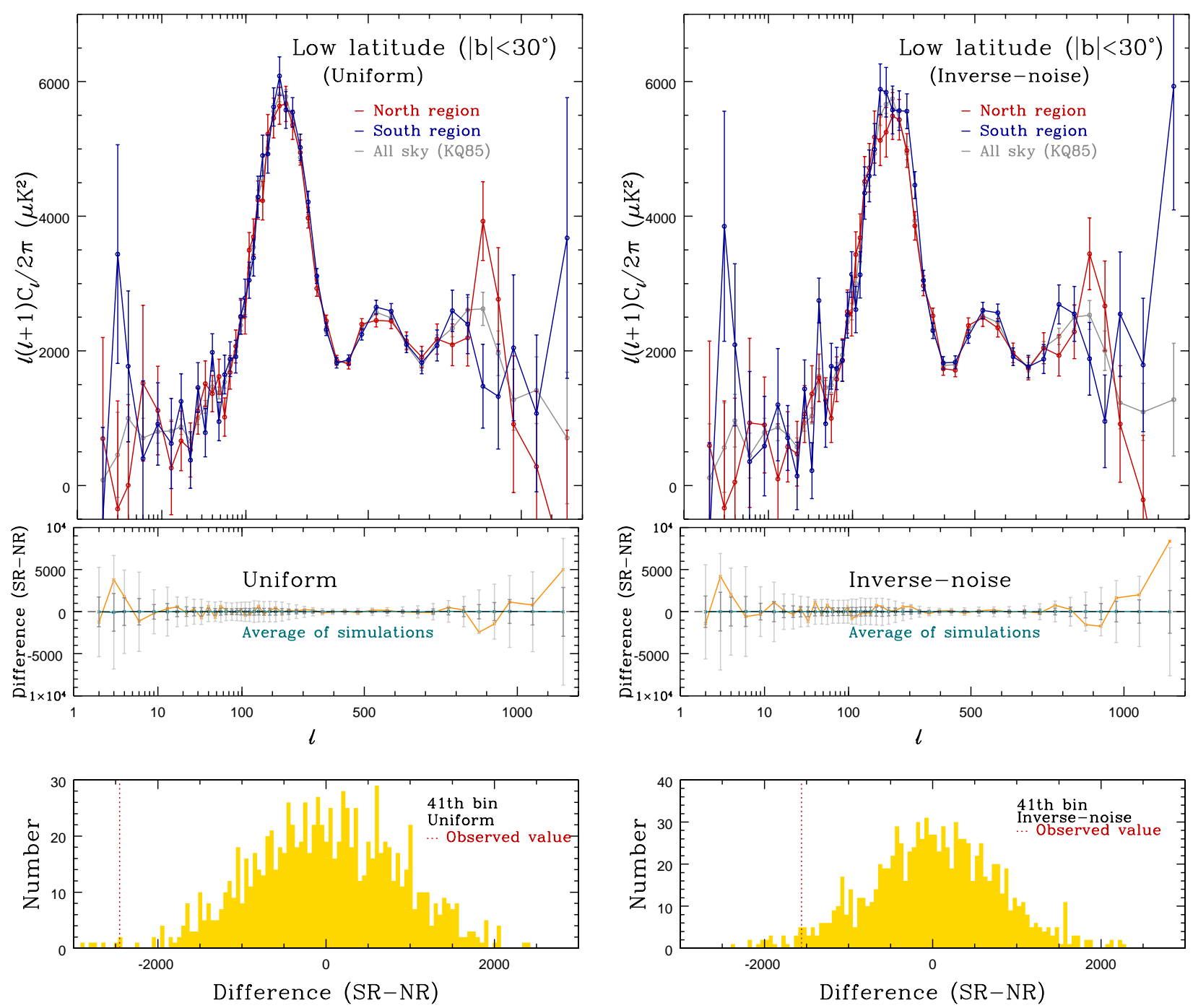

Fig. 5.- Similar to Fig. 4, but for regions in low Galactic latitude areas $\left(|b|<30^{\circ}\right)$. The power spectra are measured on the north region (NR; $0^{\circ}<b<30^{\circ}$; red) and on the south region (SR; $-30^{\circ}<b<0^{\circ}$; blue). In the bottom panels, we show histograms of power differences at the 41th $l$-bin $(851<l<900)$ between the south and north regions, expected in the concordance $\Lambda$ CDM model, together with observed values from the WMAP 7-year data (vertical red dashed lines).

around the third peak of the angular power spectrum is not due to any possible residual Galactic foreground emission.

\subsection{Instrument Noise}

Although the WMAP satellite probed the CMB temperature fluctuations on the whole sky, its scanning strategy is somewhat inhomogeneous, in the sense that the regions near the ecliptic poles were probed many times with respect to regions near the ecliptic plane. Thus, it is expected that the CMB anisotropy is strongly affected by the instrument noise in the ecliptic plane region. To quantify such an effect due to the WMAP instrument noise, we use the number of observations, $N_{\text {obs }}(p)$, at the WMAP W4 frequency channel, shown in Fig. 1.
Defining regions with high and low instrument noise by simply putting threshold limits on the number of observations gives sky regions whose boundary is not smooth and pixels near the boundary are not contiguous, which prevents us from obtaining an unbiased estimation of the power spectrum. To avoid this problem, we smooth the map of the number-of-observations at the W4 frequency channel with a Gaussian filter of $1^{\circ}$ FWHM. Then, we define the $\mathrm{NH}$ (or $\mathrm{SH}$ ) regions with high instrument noise by selecting pixels with $N_{\text {obs }}(p)<2500$ on the smoothed number-ofobservations map. We also exclude the areas not included in the primary regions. Similarly, to define regions with low instrument noise, we set $N_{\text {obs }}(p)>2035$ on the same map. The threshold value for the number of observations in the region of high (low) instru- 

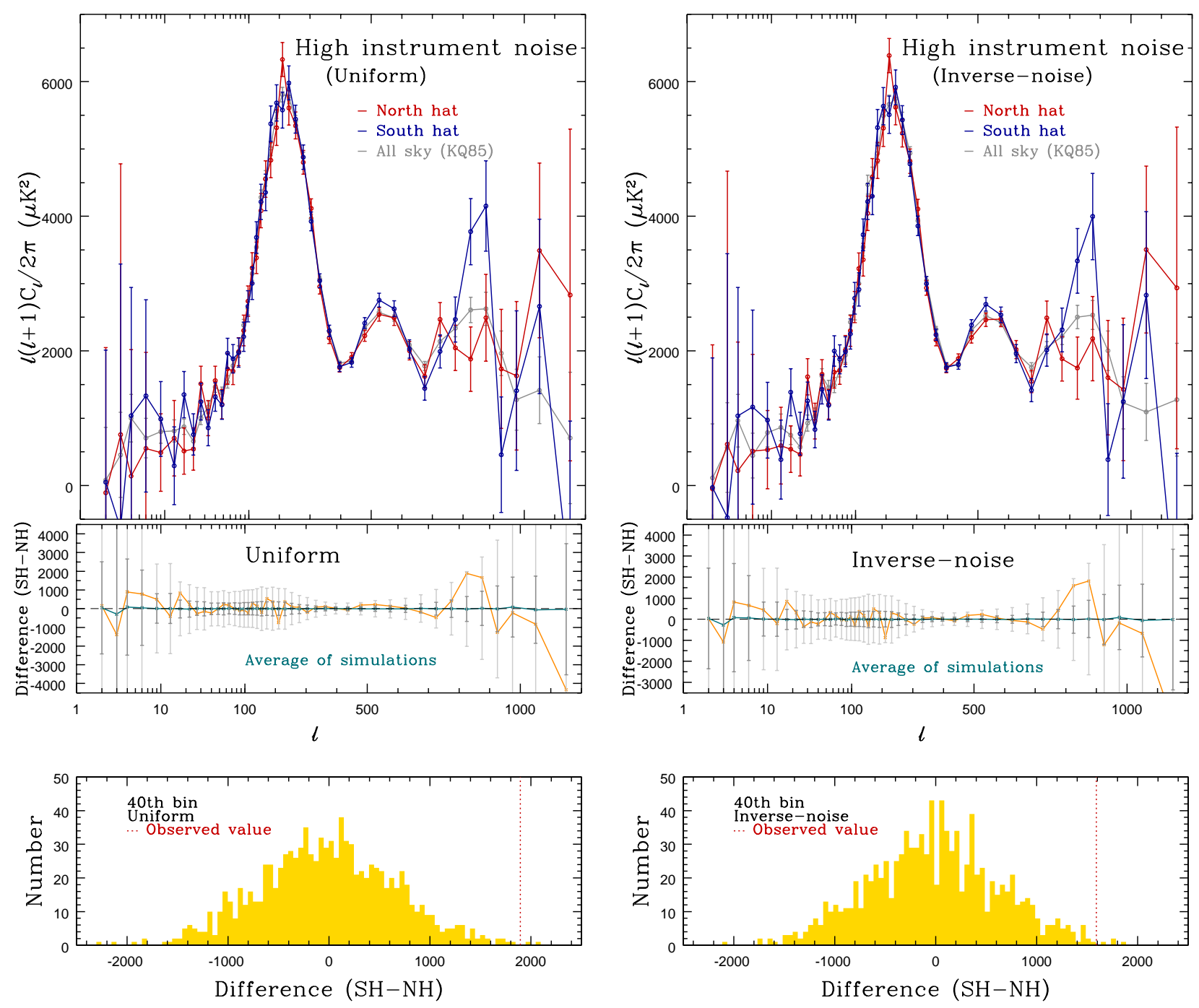

Fig. 6. - Similar to Fig. 4, but for regions in the NH (left) and SH (right) with high instrument noise $\left(N_{\text {obs }}<2500\right.$ in the W4 DA, smoothed with a FWHM $=1^{\circ}$ ).

ment noise has been set to obtain the sky area of about $15 \%(13 \%)$ of the whole sky for $N_{\text {obs }}(p)<2500$ $\left(N_{\text {obs }}(p)>2035\right)$. For ecliptic plane regions with high instrument noise (i.e., with low number of observations), we choose the threshold value to include the larger sky area for the purpose of reducing the statistical variance in the power spectrum measurement. The sky regions defined in this way are shown in Fig. $3(e)-$ $(h)$.

We have measured the angular power spectra on the ecliptic plane regions with small number of observations $\left(N_{\text {obs }}<2500\right)$ that belong to the NH or SH regions. The results are shown in Fig. 6. The angular power spectra measured on ecliptic plane regions (that belong to the $\mathrm{NH}$ or $\mathrm{SH}$ regions) show the behavior that is similar to the case of the whole $\mathrm{NH}$ and $\mathrm{SH}$ regions (see Fig. 4). The difference in the power spectrum amplitudes between the $\mathrm{SH}$ and $\mathrm{NH}$ is still statistically significant, up to $3 \sigma$.

We have also measured the angular power spectra on ecliptic pole regions with large number of observations $\left(N_{\text {obs }}(p)>2035\right)$, which are shown in Fig. 7. Here, the power spectra measured on ecliptic pole regions in the $\mathrm{NH}$ and $\mathrm{SH}$ are consistent with each other in the $l$ range around the third peak. They are also consistent with the power spectrum measured on the whole sky with the KQ85 mask (grey dots with error bars). Around the third peak, the power spectrum measured on ecliptic pole regions in the $\mathrm{NH}$ is very similar to the whole NH region, while the power spectrum measured on ecliptic pole regions in the $\mathrm{SH}$ has decreased in amplitude compared with the whole SH region (see Fig. 4). As a result, the observed north-south difference in the 40th bin of the power spectrum is around the $1 \sigma$ confidence limit and is not statistically significant any more (bottom panels of Fig. 7). These results strongly 

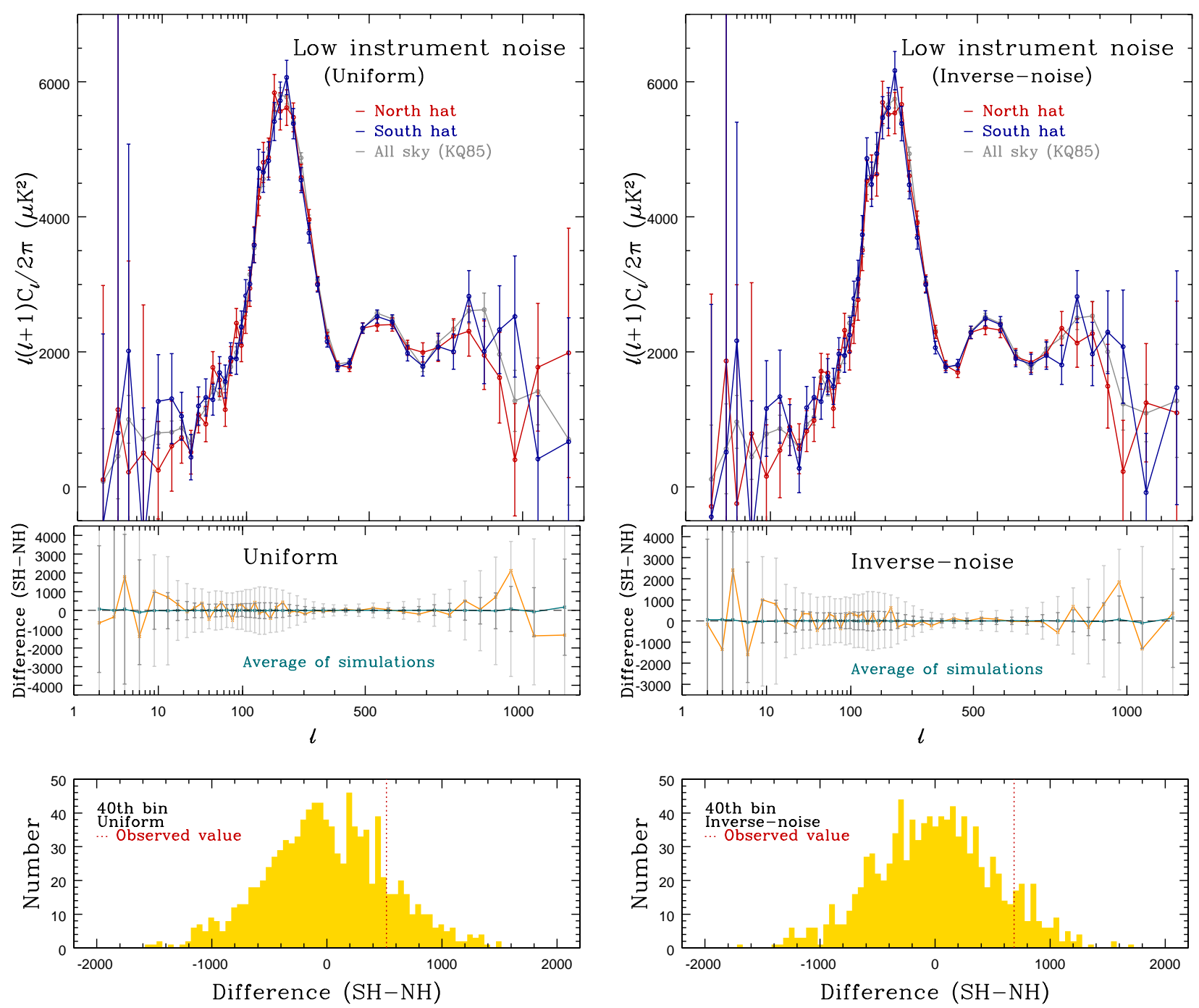

Fig. 7.- Similar to Fig. 4, but for regions with the low instrument noise $\left(N_{\mathrm{obs}}>2035\right)$ belonging to the NH (left) and SH (right).

suggest that the north-south anomaly around the third peak likely originates from the unknown systematic effects contained on sky regions affected by strong instrument noise.

Based on 1000 mocks, we count the number of cases where the magnitude of the north-south power difference is larger than the observed value for several chosen local regions. The results are summarized in Table 1 for particular $l$-bins where the high statistical significance is seen. The listed $p$-values are the probabilities of finding the north-south (absolute) difference larger than the observed value. Although the statistical significance for the north-south anomaly at high latitude regions becomes weaker for the inverse-noise weighting scheme, the observed anomaly is still rare in the $\Lambda \mathrm{CDM}$ universe with $p=0.7 \%$. The comparison with the cases of high and low instrument noise in high latitude regions strongly suggests that the north-south anomaly around the third peak comes from the region dominated by the WMAP instrument noise.

\subsection{Point Sources}

Recently, the Planck point source catalogue has been publicly available (Planck Collaboration 2011b). We take the Planck Early Release Compact Source Catalogue (ERCSC) at $100 \mathrm{GHz}$ frequency band and exclude the sources at high Galactic latitude $\left(|b| \geq 30^{\circ}\right)$ whose angular position on the sky is located in the excluded region with $M(p)=0$ in the KQ85 mask map. The remaining 163 radio point sources at $|b| \geq 30^{\circ}$ are considered as those newly discovered by the Planck satellite at $100 \mathrm{GHz}$. By excluding the circular areas centered on these point sources with radius 0.6 in the WMAP KQ85 mask map, we have produced a new mask (or window function) and measured the angular power spectra based on this mask. Because the 
Table 1.

Probabilities of finding the north-south (absolute) difference larger than the observed value, estimated based on 1000 mocks. The $l_{b}$ and $\Delta l$ symbols denote the multipole $l$-value at the center of the bin and the bin-width, respectively; they are used in the power spectrum estimation.

\begin{tabular}{cccccc}
\hline \hline area & & & & \multicolumn{2}{c}{$p$ value $(\%)$} \\
\cline { 5 - 6 } & Bin number & $l_{b}$ & $\Delta l$ & Uniform weighting & Inverse-noise weighting \\
\hline High Galactic latitude $\left(|b| \geq 30^{\circ}\right)$ & 40 & 825 & 50 & 0.1 & 0.7 \\
— with high instrument noise & 40 & 825 & 50 & 0.5 & 1.0 \\
— with low instrument noise & 40 & 825 & 50 & 31 & 0.8 \\
— with 20\% increased bin-width & 39 & 825 & 60 & 0.0 & 2.5 \\
— with 50\% increased bin-width & 39 & 825 & 75 & 0.0 & 5.9 \\
\hline High Galactic latitude $\left(|b| \geq 25^{\circ}\right)$ & 40 & 825 & 50 & 0.6 & 0.0 \\
High Galactic latitude $\left(|b| \geq 35^{\circ}\right)$ & 40 & 825 & 50 & 0.0 & 1.1 \\
High Galactic latitude $\left(|b| \geq 40^{\circ}\right)$ & 40 & 825 & 50 & 1.5 & 12 \\
High Galactic latitude $\left(|b| \geq 45^{\circ}\right)$ & 40 & 825 & 50 & 20 & 5.0 \\
\hline Low Galactic latitude $\left(|b|<30^{\circ}\right)$ & 41 & 875 & 50 & 0.5 & 0.1 \\
\hline \hline
\end{tabular}

number of newly discovered sources is very small, the additional exclusion of point sources almost does not affect the angular power spectra on the $\mathrm{NH}$ and $\mathrm{SH}$ regions. Therefore, at the present stage we cannot find any evidence that the north-south anomaly around the third peak of the angular power spectrum originates from unresolved point sources.

\subsection{Dependence of the North-South Anomaly on the Bin-Width and on the Galactic Lat- itude Cut}

Here we investigate the effect of the bin-width and of the Galactic latitude cut on the detected north-south anomaly around the third peak in the angular power spectrum measured from the WMAP 7-year data.

Our detection of the anomaly is based on the 40th bin around the third peak, which ranges over $851 \leq$ $l \leq 900(\Delta l=50)$; see Fig. 4 . By fixing the bin center at $l=825$, we have increased the bin-width by $20 \%$ $(796 \leq l \leq 855 ; \Delta l=60)$ and $50 \%(788 \leq l \leq 862$; $\Delta l=75)$. During the re-binning process, we reduce the total number of bins to 43 and enlarge the width of adjacent bins appropriately; now the bin centered at $l=825$ is located at the 39 th bin. Based on the new $l$-binning, we have measured angular power spectra from the WMAP 7-year data and mock observations. The results are shown in Fig. 8. In the case of uniform weighting scheme, the larger bin-width increases the statistical significance of the north-south anomaly; there is no such an anomaly larger than the observed one among the 1000 WMAP simulations. However, in the case of inverse-noise weighting scheme, the statistical significance slightly decreases for the $50 \%$ increased bin-width (see Table 1 for a summary of statistical significance).

We also check if our conclusion is sensitive to the
Galactic latitude cut. We compare the power spectrum measurements for different latitude cuts, $|b|=25^{\circ}, 30^{\circ}$, $35^{\circ}, 40^{\circ}$, and $45^{\circ}$ (Fig. 9; the case of $|b|=30^{\circ}$ is presented in Fig. 4). We notice that the north-south anomaly becomes significant in the case of $|b|=35^{\circ}$ cut, and no anomaly occurs, in both weighting schemes, among the mock data sets (see Table 1). Furthermore, the statistical significance of this anomaly becomes weaker as the latitude cut is higher or lower than $|b|=35^{\circ}$. For $|b|=25^{\circ}\left(45^{\circ}\right)$, the $p$ value is $5.9 \%(12 \%)$ in the inverse-noise weighting scheme. Up to the Galactic latitude cut $|b|=40^{\circ}$, the north-south anomaly is maintained with high statistical significance (see Table 1 ). For higher latitude cuts like $|b| \geq 45^{\circ}$, however, the north-south anomaly in the power spectrum amplitude does not have any statistical significance.

According to the two results shown above, the observed north-south anomaly has weak dependences on the bin-width used in the power spectrum estimation and the Galactic latitude cut, which implies that it is a realistic feature present in the WMAP data and supports our primary conclusion.

\section{CONCLUSIONS}

In this work we have compared the angular power spectra measured on various local sky regions using the WMAP 7-year temperature anisotropy data. The areas studied in this work are the low Galactic contamination regions at high Galactic latitude ( $\mathrm{NH}$ and $\mathrm{SH})$, the strong Galactic contamination regions at low Galactic latitude, the regions dominated by the WMAP instrument noise (ecliptic plane regions), and those of low instrument noise (ecliptic pole regions).

We found that the power spectra around the third peak measured on the $\mathrm{NH}$ and $\mathrm{SH}$ regions show an 

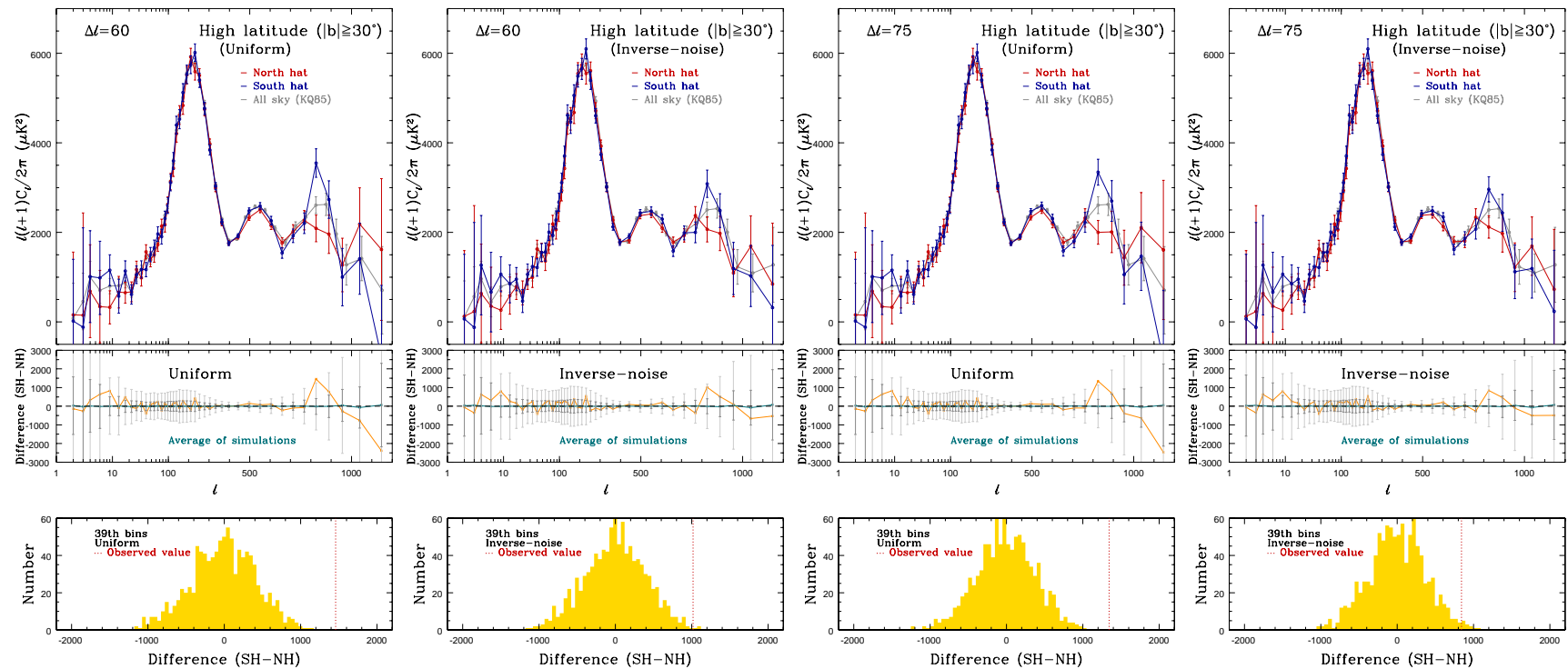

Fig. 8. - Similar to Fig. 4, but now the bin-width has been increased by a factor of 1.2 (first two panels) and 1.5 (last two panels). The 39th bin centered at $l=825$ corresponds to the bin located around at the third peak with the bin ranging over $796 \leq l \leq 855(\Delta l=60)$ for $20 \%$ increased bin-width and $788 \leq \ell \leq 862(\Delta l=75)$ for $50 \%$ increased one. The results obtained in the uniform weighting scheme are shown on the first and third panels, while those in the inverse-noise weighting scheme on the second and fourth ones. In both cases, the high Galactic latitude regions with $|b| \geq 30^{\circ}$ have been used. For the power spectrum measured on the whole sky enclosed by the KQ85 mask (grey color), the original $l$-binning has been used as in Fig. 4.

anomaly statistically significant, around $3 \sigma$ from the $\Lambda \mathrm{CDM}$ model prediction. We have tried to identify the cause of this anomaly by performing a similar analysis on the low latitude regions and in regions with high or low instrument noise. Curiously, at low Galactic latitude $\left(|b|<30^{\circ}\right)$ there appears another but less statistically significant north-south anomaly around the third peak, whose behavior is opposite to the one seen in high latitude regions and compensates the anomalies in the whole northern and southern hemispheres. At the present time, we cannot draw any firm conclusion about the origin of the observed anomaly. However, we found that the anomaly remains, with high statistical significance, in the power spectra measured on regions with high instrument noise, and becomes weaker on regions with low instrument noise. Thus, in our present analysis the observed anomaly is significant on sky regions dominated by WMAP instrument noise. We have also verified that it has only weak dependences on the bin-width used in the power spectrum estimation, and on the Galactic latitude cut, which support our conclusion (see Figs. 8 and 9).

The location of the third peak in the angular power spectrum corresponds to the angular scale that approaches the WMAP resolution limit and that is dominated by WMAP instrument noise. Because the Planck satellite probes the CMB anisotropy with higher angular resolution and sensitivity than WMAP, we expect that the origin of the observed anomaly will be identified in more detail when the Planck data becomes publicly available (Planck Collaboration 2011a).

It is possible that our detection of the north-south anomaly may be strongly driven by a posteriori statistics, based on the fact that we have computed the power spectra on two different parts of the sky and only then noticed a peculiar discrepancy between the two power amplitudes in one bin around the third peak, neglecting that there could have been a similar discrepancy in any of the other multipole bins (see Zhang \& Huterer 2010 for a related discussion). One may argue that we should properly quantify the statistical significance of the detected anomaly from the distribution of the maximum north-south differences (in unit of the standard deviation) over all the multipole bins. The result for the $\mathrm{NH}$ and $\mathrm{SH}\left(|b| \geq 30^{\circ}\right)$ in the uniform weighting scheme is shown in Fig. 10. In the histogram of 1000 values of the maximum (among 45 bins) northsouth power difference in unit of the standard deviation, each estimated from WMAP mock observations, the detected anomaly becomes less statistically significant; the probability of finding cases with a deviation larger than the measured value is $p=75 / 1000$ (i.e., $7.5 \%$ ) which is slightly less than $2 \sigma$.

However, it should be emphasized that what described above is correct only under the condition that the power spectrum at different multipoles is equally affected by the same CMB physics, Galaxy foreground, and instrument noise properties, which is generally not true in harmonic space. The power spectrum at low multipoles is dominated by the integrated Sachs-Wolfe 

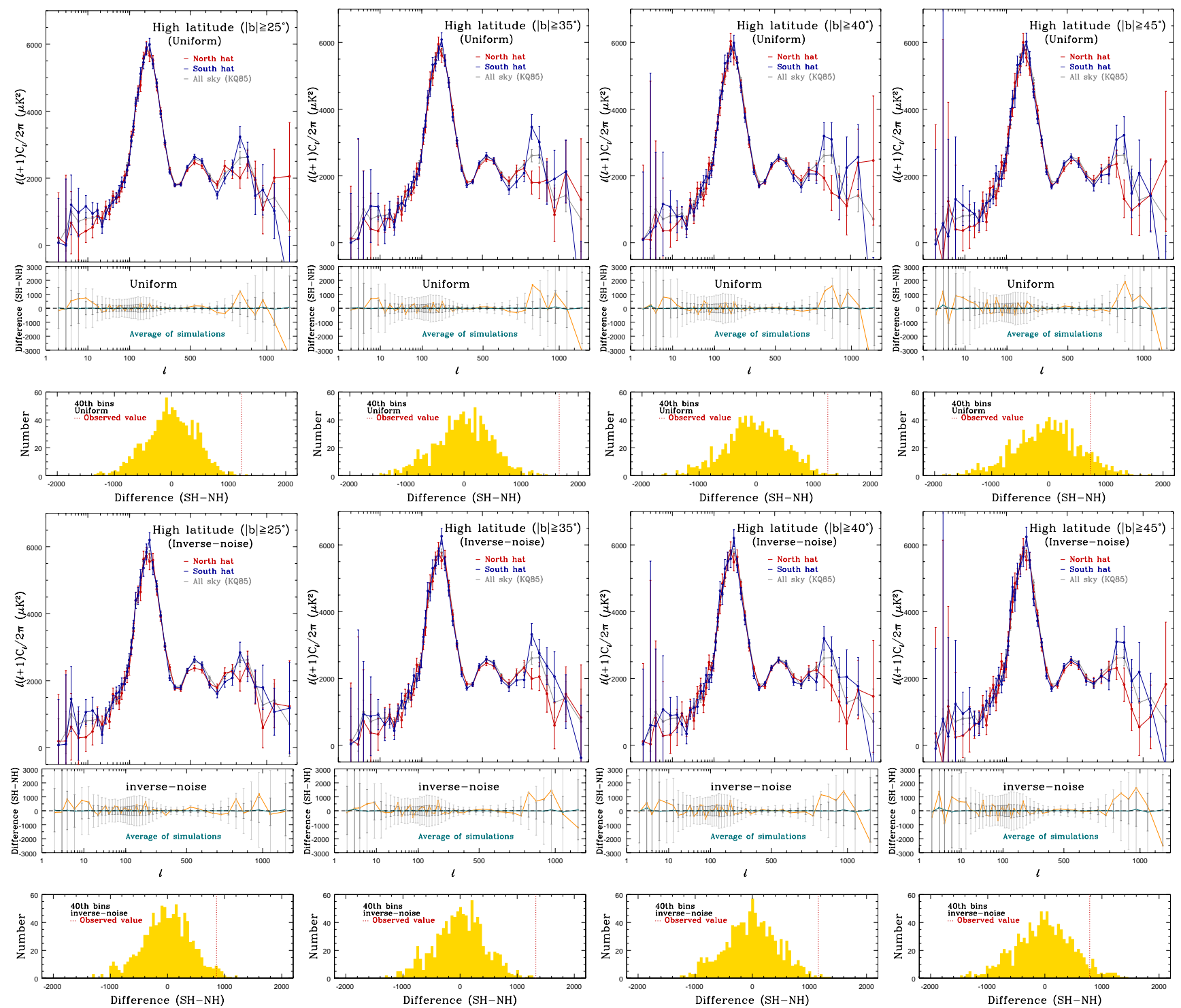

Fig. 9.- Angular power spectra measured on the $\mathrm{NH}$ and $\mathrm{SH}$ regions defined by different Galactic latitude cuts (from left to right, $|b|=25^{\circ}, 35^{\circ}, 40^{\circ}, 45^{\circ}$ ), with a similar format given in Fig. 4 (the case of $|b|=30^{\circ}$ ). Fractions of the sky area for north (south) regions are $27.7 \%(27.6 \%), 20.6 \%$ (20.5\%), 17.2\% (17.3\%), 14.1\% (14.2\%), respectively, in increasing order of latitude cut. Top panels are for the uniform weighting scheme, while bottom panels for the inverse-noise weighting scheme.

effect, and more affected by the survey geometry, while the power spectrum at higher multipoles is related to the physics of acoustic oscillations and WMAP instrument noise. For example, it is not justified to consider all the multipole bins in quantifying the statistical significance of an anomaly detected in one multipole bin, if the anomaly originates from a phenomenon that affects only the local multipole range. Therefore, our original interpretation of the detected north-south anomaly around the third peak as statistically significant (based on the analysis of the corresponding multipole bin) is still valid before the origin of the anomaly becomes unveiled.
As Planck data will soon be available, we anticipate that our issue of whether or not the anomaly is intrinsic or due to WMAP instrument noise will be resolved.

\section{ACKNOWLEDGMENTS}

We acknowledge the use of the Legacy Archive for Microwave Background Data Analysis (LAMBDA). Support for LAMBDA is provided by the NASA Office of Space Science. Some of the results in this paper have been derived using the HEALPix and CAMB software packages. This work was supported by the Korea Re- 


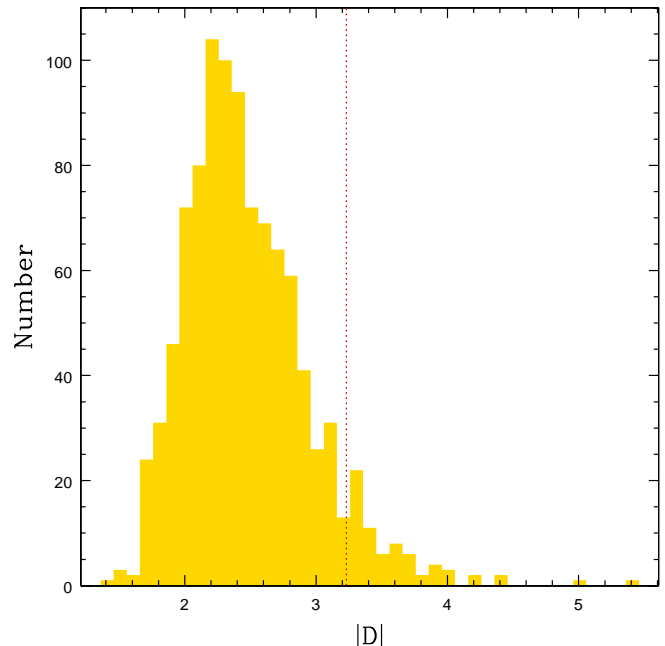

Fig. 10. - Histogram of 1000 values of the maximum (among 45 bins) north-south power difference in units of standard deviation, $|D|=\operatorname{Max}\left(\left|C_{b}^{\mathrm{NH}}-C_{b}^{\mathrm{SH}}\right| / \sigma_{b}\right) ; C_{b}^{\mathrm{NH}}$ $\left(C_{b}^{\mathrm{SH}}\right)$ is the $b$-th band power measured at the north (south) hat region $\left(|b| \geq 30^{\circ}\right)$, and $\sigma_{b}$ is the standard deviation of the power difference at the $b$-th multipole bin estimated from the WMAP simulation data sets, equivalent to the size of the dark grey $(1 \sigma)$ error bars in the middle-left panel of Fig. 4. The vertical red dashed line indicates the measured value with $|D|=3.23$.

search Foundation Grant funded by the Korean Government (KRF-2008-341-C00022).

\section{REFERENCES}

Alpher, R. A., \& Herman, R. 1948, Evolution of the Universe, Nature, 162, 774

Ansari, R., \& Magneville, C. 2010, Partial CMB Maps: Bias Removal and Optimal Binning of the Angular Power Spectrum, MNRAS, 405, 1421

Basak, S., \& Delabrouille, J. 2012, A Needlet Internal Linear Combination Analysis of WMAP 7-Year Data: Estimation of CMB Temperature Map and Power Spectrum, MNRAS, 419, 1163

Benoît, A., Ade, P., Amblard, A., et al. 2003, The Cosmic Microwave Background Anisotropy Power Spectrum Measured by Archeops, A\&A, 399, L19

Bennett, C. L., Halpern, M., Hinshaw, G., et al. 2003, First-Year Wilkinson Microwave Anisotropy Probe (WMAP) Observations: Preliminary Maps and Basic Results, ApJS, 148, 1

Bernui, A. 2008, Anomalous CMB North-South Asymmetry, Phys. Rev. D, 78, 063531

Bond, J. R., \& Efstathiou, G. 1987, The Statistics of Cosmic Background Radiation Fluctuations, MNRAS, 226, 655
Bond, J. R., Jaffe, A. H., \& Knox, L. 1998, Estimating the Power Spectrum of the Cosmic Microwave Background, Phys. Rev. D, 57, 2117

Brown, M. L., Castro, P. G., \& Taylor, A. N. 2005, Cosmic Microwave Background Temperature and Polarization Pseudo- $C_{l}$ Estimators and Covariances, MNRAS, 360, 1262

Brown, M. L., Ade, P., Bock, J., et al. 2009, Improved Measurements of the Temperature and Polarization of the Cosmic Microwave Background from QUaD, ApJ, 705, 978

Carlstrom, J. E., Kovac, J., Leitch, E. M., \& Pryke, C. 2003, Status of CMB Polarization Measurements from DASI and Other Experiments, New Astron. Rev., 47, 953

Chiang, L. Y., \& Chen, F. F. 2012, Direct Measurement of the Angular Power Spectrum of Cosmic Microwave Background Temperature Anisotropies in the WMAP Data, ApJ, 751, 43

Chon, G., Challinor, A., Prunet, S., Hivon, E., \& Szapudi, I. 2004, Fast Estimation of Polarization Power Spectra Using Correlation Functions, MNRAS, 350 , 914

Dahlen, F. A., \& Simons, F. J. 2008, Spectral Estimation on a Sphere in Geophysics and Cosmology, Geophysical Journal International, 174, 774

Das, S., Hajian, A., \& Spergel, D. N. 2009, Efficient Power Spectrum Estimation for High Resolution CMB Maps, Phys. Rev. D, 79, 083008

Dicke, R. H., Peebles, P. J. E., Roll, P. G., \& Wilkinson, D. T. 1965, Cosmic Black-Body Radiation, ApJ, 142,414

Efstathiou, G. 2004, Myths and Truths Concerning Estimation of Power Spectra: the Case for a Hybrid Estimator, MNRAS, 349, 603

Eriksen, H. K., O'Dwyer, I. J., Jewell, J. B., et al. 2004a, Power Spectrum Estimation from HighResolution Maps by Gibbs Sampling, ApJS, 155, 227

Eriksen, H. K., Hansen, F. K., Banday, A. J., Górski, K. M., \& Lilje, P. B. 2004b, Asymmetries in the Cosmic Microwave Background Anisotropy Field, ApJ, 605,14

Eriksen, H. K., Novikov, D. I., Lilje, P. B., Banday, A. J., \& Górski, K. M. 2004c, Testing for NonGaussianity in the Wilkinson Microwave Anisotropy Probe Data: Minkowski Functionals and the Length of the Skeleton, ApJ, 612, 64

Eriksen, H. K., Huey, G., Saha, R., et al. 2007a, A Reanalysis of the 3 Year Wilkinson Microwave Anisotropy Probe Temperature Power Spectrum and Likelihood, ApJ, 656, 641

Eriksen, H. K., Banday, A. J., Górski, K. M., Hansen, F. K., \& Lilje, P. B. 2007b, Hemispherical Power Asymmetry in the Third-Year Wilkinson Microwave Anisotropy Probe Sky Maps, ApJ, 660, L81 
Faÿ, G., Guilloux, F., Betoule, M., et al. 2008, CMB Power Spectrum Estimation Using Wavelets, Phys. Rev. D, 78, 083013

Fowler, J. W., Acquaviva, V., Ade, P. A. R., et al. 2010, The Atacama Cosmology Telescope: A Measurement of the $600<\ell<8000$ Cosmic Microwave Background Power Spectrum at 148 GHz, ApJ, 722, 1148

Górski, K. M. 1994, On Determining the Spectrum of Primordial Inhomogeneity from the COBE DMR Sky Maps: Method, ApJ, 430, L85

Górski, K. M., Wandelt, B. D., Hansen, F. K., Hivon, E., \& Banday, A. J. 1999, The HEALPix Primer, arXiv:astro-ph/9905275

Górski, K. M., Hivon, E., Banday, A. J., et al. 2005, HEALPix: A Framework for High-Resolution Discretization and Fast Analysis of Data Distributed on the Sphere, ApJ, 622, 759

Hansen, F. K., Górski, K. M., \& Hivon, E. 2002, Gabor Transforms on the Sphere with Applications to CMB Power Spectrum Estimation, MNRAS, 336, 1304

Hansen, F. K., Banday, A. J., \& Górski, K. M. 2004a, Testing the Cosmological Principle of Isotropy: Local Power-Spectrum Estimates of the WMAP Data, MNRAS, 354, 641

Hansen, F. K., Balbi, A., Banday, A. J., \& Górski, K. M. 2004b, Cosmological Parameters and the WMAP Data Revisited, MNRAS, 354, 905

Hansen, F. K., Banday, A. J., Górski, K. M., Eriksen, H. K., \& Lilje, P. B. 2009, Power Asymmetry in Cosmic Microwave Background Fluctuations from Full Sky to Sub-Degree Scales: Is the Universe Isotropic?, ApJ, 704, 1448

Hinshaw, G., Spergel, D. N., Verde, L., et al. 2003, First-Year Wilkinson Microwave Anisotropy Probe (WMAP) Observations: The Angular Power Spectrum, ApJS, 148, 135

Hinshaw, G., Nolta, M. R., Bennett, C. L., et al. 2007, Three-Year Wilkinson Microwave Anisotropy Probe (WMAP) Observations: Temperature Analysis, ApJS, 170, 288

Hivon, E., Górski, K. M., Netterfield, C. B., et al. 2002, MASTER of the Cosmic Microwave Background Anisotropy Power Spectrum: A Fast Method for Statistical Analysis of Large and Complex Cosmic Microwave Background Data Sets, ApJ, 567, 2

Hoftuft, J., Eriksen, H. K., Banday, A. J., et al. 2009, Increasing Evidence for Hemispherical Power Asymmetry in the Five-Year WMAP Data, ApJ, 699, 985

Hu, W., \& Dodelson, S. 2002, Cosmic Microwave Background Anisotropies, ARA\&A, 40, 171

Huffenberger, K. M., Eriksen, H. K., \& Hansen, F. K. 2006, Point-Source Power in 3 Year Wilkinson Microwave Anisotropy Probe Data, ApJ, 651, L81
Jarosik, N., Bennett, C. L., Dunkley, J., et al. 2011, Seven-Year Wilkinson Microwave Anisotropy Probe (WMAP) Observations: Sky Maps, Systematic Errors, and Basic Results, ApJS, 192, 14

Jones, W. C., Ade, P. A. R., Bock, J. J., et al. 2006, A Measurement of the Angular Power Spectrum of the CMB Temperature Anisotropy from the 2003 Flight of BOOMERANG, ApJ, 647, 823

Keisler, R., Reichardt, C. L., Aird, K. A., et al. 2011, A Measurement of the Damping Tail of the Cosmic Microwave Background Power Spectrum with the South Pole Telescope, ApJ, 743, 28

Komatsu, E., Dunkley, J., Nolta, M. R., et al. 2009, Five-Year Wilkinson Microwave Anisotropy Probe Observations: Cosmological Interpretation, ApJS, 180,330

Komatsu, E., Smith, K. M., Dunkley, J., et al. 2011, Seven-Year Wilkinson Microwave Anisotropy Probe (WMAP) Observations: Cosmological Interpretation, ApJS, 192, 18

Larson, D., Dunkley, J., Hinshaw, G., et al. 2011, Seven-Year Wilkinson Microwave Anisotropy Probe (WMAP) Observations: Power Spectra and WMAP-derived Parameters, ApJS, 192, 16

Lee, A. T., Ade, P., Balbi, A., et al. 2001, A High Spatial Resolution Analysis of the MAXIMA-1 Cosmic Microwave Background Anisotropy Data, ApJ, 561, L1

Lewis, A., Challinor, A., \& Lasenby, A. 2000, Efficient Computation of Cosmic Microwave Background Anisotropies in Closed FriedmannRobertson-Walker Models, ApJ, 538, 473

Mason, B. S., Pearson, T. J., Readhead, A. C. S., et al. 2003, The Anisotropy of the Microwave Background to $l=3500$ : Deep Field Observations with the Cosmic Background Imager, ApJ, 591, 540

Mitra, S., Sengupta, A. S., Ray, S., Saha, R., \& Souradeep, T. 2009, Cosmic Microwave Background Power Spectrum Estimation with NonCircular Beam and Incomplete Sky Coverage, MNRAS, 394, 1419

Mortlock, D. J., Challinor, A. D., \& Hobson, M. P. 2002, Analysis of Cosmic Microwave Background Data on an Incomplete Sky, MNRAS, 330, 405

Nolta, M. R., Dunkley, J., Hill, R. S., et al. 2009, FiveYear Wilkinson Microwave Anisotropy Probe Observations: Angular Power Spectra, ApJS, 180, 296

Oh, S. P., Spergel, D. N., \& Hinshaw, G. 1999, An Efficient Technique to Determine the Power Spectrum from Cosmic Microwave Background Sky Maps, ApJ, 510, 551

Page, L., Nolta, M. R., Barnes, C., et al. 2003, First-Year Wilkinson Microwave Anisotropy Probe (WMAP) Observations: Interpretation of the TT and TE Angular Power Spectrum Peaks, ApJS, 148, 233 
Page, L., Hinshaw, G., Komatsu, E., et al. 2007, Three-Year Wilkinson Microwave Anisotropy Probe (WMAP) Observations: Polarization Analysis, ApJS, 170, 335

Park, C.-G. 2004, Non-Gaussian Signatures in the Temperature Fluctuation Observed by the Wilkinson Microwave Anisotropy Probe, MNRAS, 349, 313

Peebles, P. J. E., \& Yu, J. T. 1970, Primeval Adiabatic Perturbation in an Expanding Universe, ApJ, 162, 815

Peiris, H. V., Komatsu, E., Verde, L., et al. 2003, First-Year Wilkinson Microwave Anisotropy Probe (WMAP) Observations: Implications For Inflation, ApJS, 148, 213

Penzias, A. A., \& Wilson, R. W. 1965, A Measurement of Excess Antenna Temperature at 4080 Mc/s, ApJ, 142,419

Planck Collaboration, Ade, P. A. R., Aghanim, N., et al. 2011a, Planck Early Results. I. The Planck Mission, A\&A, 536, A1

Planck Collaboration, Ade, P. A. R., Aghanim, N., et al. 2011, Planck Early Results. VII. The Early Release Compact Source Catalogue, A\&A, 536, A7

Polenta, G., Marinucci, D., Balbi, A., et al. 2005, Unbiased Estimation of an Angular Power Spectrum, J. Cosmol. Astropart. Phys., 11, 1

Reichardt, C. L., Ade, P. A. R., Bock, J. J., et al. 2009, High-Resolution CMB Power Spectrum from the Complete ACBAR Data Set, ApJ, 694, 1200

Sachs, R. K., \& Wolfe, A. M. 1967, Perturbations of a Cosmological Model and Angular Variations of the Microwave Background, ApJ, 147, 73

Saha, R., Jain, P., \& Souradeep, T. 2006, A Blind Estimation of the Angular Power Spectrum of CMB Anisotropy from WMAP, ApJ, 645, L89

Saha, R., Prunet, S., Jain, P., \& Souradeep, T. 2008, CMB Anisotropy Power Spectrum Using Linear Combinations of WMAP Maps, Phys. Rev. D, 78, 023003

Samal, P. K., Saha, R., Delabrouille, J., et al. 2010, Cosmic Microwave Background Polarization and Temperature Power Spectra Estimation Using Linear Combination of WMAP 5 Year Maps, ApJ, 714, 840

Scott, D., \& Smoot, G. 2006, Cosmic Microwave Background Mini-Review, arXiv:astro-ph/0601307

Smoot, G. F., Bennett, C. L., Kogut, A., et al. 1992, Structure in the COBE Differential Microwave Radiometer First-Year Maps, ApJ, 396, L1

Souradeep, T., Saha, R., \& Jain, P. 2006, Angular Power Spectrum of CMB Anisotropy from WMAP, New Astron. Rev., 50, 854
Spergel, D. N., Verde, L., Peiris, H. V., et al. 2003, First-Year Wilkinson Microwave Anisotropy Probe (WMAP) Observations: Determination of Cosmological Parameters, ApJS, 148, 175

Spergel, D. N., Bean, R., Doré, O., et al. 2007, Three-Year Wilkinson Microwave Anisotropy Probe (WMAP) Observations: Implications for Cosmology, ApJS, 170, 377

Szapudi, I., Prunet, S., Pogosyan, D., Szalay, A. S., \& Bond, J. R. 2001, Fast Cosmic Microwave Background Analyses via Correlation Functions, ApJ, 548, L115

Tegmark, M. 1997, How to Measure CMB Power Spectra without Losing Information, Phys. Rev. D, 55, 5895

Wandelt, B. D., Hivon, E., \& Górski, K. M. 2001, Cosmic Microwave Background Anisotropy Power Spectrum Statistics for High Precision Cosmology, Phys. Rev. D, 64, 083003

Wandelt, B. D., \& Hansen, F. K. 2003, Fast, Exact CMB Power Spectrum Estimation for a Certain Class of Observational Strategies, Phys. Rev. D, 67, 023001

Wandelt, B. D., Larson, D. L., \& Lakshminarayanan, A. 2004, Global, Exact Cosmic Microwave Background Data Analysis Using Gibbs Sampling, Phys. Rev. D, 70, 083511

Yoho, A., Ferrer, F., \& Starkman, G. D. 2011, DegreeScale Anomalies in the CMB: Localizing the First Peak Dip to a Small Patch of the North Ecliptic Sky, Phys. Rev. D, 83, 083525

Zhang, R., \& Huterer, D. 2010, Disks in the Sky: A Reassessment of the WMAP "Cold Spot"?, Astroparticle Physics, 33, 69 